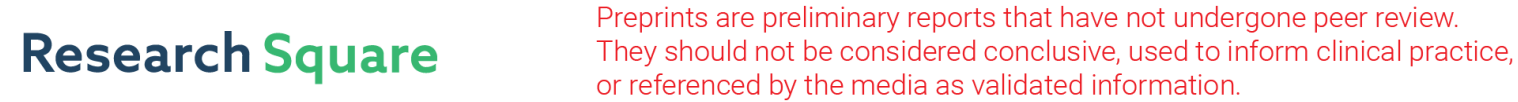 \\ Clinical guidelines reduce unnecessary preoperative echocardiograph in elderly hip fracture patients
}

\section{Xuepan Chen}

Guangdong General Hospital

Yuanchen $\mathrm{Ma}$

Guangdong General Hospital

Qingtian Li

Guangdong General Hospital

Zhantao Deng

Guangdong General Hospital

JunXing Liao

Guangdong General Hospital

Qiujian Zheng ( $\nabla$ zqj106@126.com )

Guangdong General Hospital

\section{Research article}

Keywords: clinical guidelines, elderly, hip fracture, preoperative cardiac test

Posted Date: February 27th, 2020

DOl: https://doi.org/10.21203/rs.2.24705/v1

License: (c) (1) This work is licensed under a Creative Commons Attribution 4.0 International License.

Read Full License 


\section{Abstract}

Background The American College of Cardiology (ACC) and the American Heart Association (AHA) have set up guidelines for preoperative usage of transthoracic echocardiograph (TTE) in case of non-cardiac surgery. Unnecessary TTE would increase hospital cost and delay surgery. Therefore, we aimed to analyze whether the TTE before hip fracture surgery was in accordance with guidelines and what the effects were on outcome.

Methods 281 elderly patients who had surgery for an isolated hip fracture were involved. The preoperative TTE test and adherence to the guideline were analyzed. TTE reports were reviewed for the presence of major cardiac abnormality with potentiality to change perioperative management, including left ventricular systolic or diastolic dysfunction, pericardial effusion, moderate or severe valvular disease, and pulmonary hypertension. Primary outcome was time to surgery (TTS), length of hospital stay (LLS), postoperative complications. Secondary outcome was sensitivity and specificity of accordance with the guideline for identifying which patients would have TTE that demonstrated major cardiac abnormality.

Results Of the 281 patients included, 174 patients (61.9\%) received the TTE, whereas 107 patients did not. Only 71 of the 174 patients (40.8\%) with TTE met the guidelines. Among the 202 patients who did not meet the guidelines (103 with TTE and 99 without TTE), those who received a preoperative TTE had a longer TTS (4.24 vs. 2.10 days, $P=0.000)$ and LOS (10.44 vs. 7.72 days, $P=0.000)$. There were no differences in postoperative complication, the sensitivity and specificity of guidelines as the screening tool to identify patients with major cardiac abnormality was $86.7 \%$ and $68.7 \%$.

Conclusion Preoperative TTE is frequently overused out of guidelines and lead to unnecessary surgery delay while not reducing the rate of postoperative complications. Stricter adherence to guidelines should be recommended, as it would help decrease unnecessary test.

\section{Background}

Hip fracture is the major reason of hospital admission in elderly patients. With a constantly increasing incidence, it is estimated to reach 6.3 million worldwide by the year 2050[1]. Elderly patients with hip fracture commonly presented with multiple comorbidities associated with high morbidity and mortality rates[2, 3]. Cardiovascular complications were the principal causes of death after hip fracture[4, 5]. On the one hand, early surgical intervention is commonly recommended for hip fracture patients as the surgery delay is associated with increased complications and mortality [6, 7]. On the other hand, early surgery is often delayed due to preoperative cardiac risk assessment and stratification. This may involve the preoperative transthoracic echocardiography (TTE) as it is often time consuming. Therefore, extended preoperative TTE should be restricted if it is unlikely to identify the major cardiac abnormality or change perioperative patient management. In 2014, the American College of Cardiology (ACC) Foundation and the American Heart Association (AHA) developed guidelines to determine which patients require preoperative TTE in non-cardiac surgery[8]. The guidelines aimed to identify patients who were most 
likely to demonstrate major cardiac abnormality on TTE and consequently reduce unnecessary test. Despite the existence of guidelines, the gap between guideline and clinical practice often exists[9]. In addition, the appropriateness of this guidelines in the management of elderly patients with hip fractures was not established. Therefore, we aimed to identify how often preoperative transthoracic echocardiograph (TTE) is made in accordance with the guidelines and the influence caused by the unnecessary TTE. In addition, to assess whether the guidelines decrease TTE without missing the important cardiac disease.

\section{Patients And Methods}

\section{Study population}

After institutional review board approval, we retrospectively reviewed the electronic medical records of surgically treated hip fractures (femoral neck, intertrochanteric, sub-trochanteric) in elderly patients from January 1, 2015 to January 1, 2018. Exclusion criteria including (1) multiple fractures besides hip fracture, (2) pathological hip fracture or patients with malignant disease, (3) periprosthetic fracture, (4) surgery delay not related to echocardiography test (e.g. anticoagulant withdrawal, unavailable operation room).

The parameters extracted from medical records included age, sex, fracture type, surgery type, American Society of Anesthesiologists (ASA) class, anesthesiology type, preoperative comorbidities, postoperative complications, TTE reports, date and time of admission, date of echocardiography, surgery and discharged.

\section{Whether adhere to guideline or not}

For each patient included, charts were reviewed whether it was recommended to undergo TTE or not according to ACC/AHA guidelines. The indications for undergoing TTE included (1) dyspnea of unknown origin, (2) worsening of known signs or symptoms of heart failure, (3) known history of valvular dysfunction or heart failure without TTE in the last year or worsened symptoms, and (4) suspicion of moderate or greater valvular stenosis or regurgitation.[8] If the patient met one or more of the indications above, it was determined that the patient should undergo TTE. As a result, the patients were divided into 4 groups, including patients met the ACC/AHA guideline and underwent TTE; patients did not meet the guideline but undergo TTE; patients met the guideline but did not undergo TTE; patients did not meet the guideline or undergo TTE.

\section{Major cardiac abnormality}

Additional analyses with the subgroup of patients with a preoperative TTE was conducted. The preoperative TTE report was reviewed to identify whether there was major cardiac abnormality with potentiality to change perioperative management. According to previous literature, these conditions included a left ventricular ejection fraction diminished by $<25 \%$, systolic pulmonary artery hypertension 
$>55 \mathrm{~mm} \mathrm{Hg}$, "moderate" or "severe" valvular disease and pericardial effusion.[10-12] The TTE was defined to have the potentiality to change management if it had one or more of major cardiac abnormalities listed above.

\section{Statistical analysis}

Continuous variables are expressed as mean \pm standard deviation and compared using Student's $t$ test between those who did not meet the guideline but underwent TTE and those who did not meet the guideline or undergo TTE. Categorical variables are expressed as percentages and compared using the Chi-squared test or Fisher exact test as appropriate between 2 groups. The assessment of the ACC/AHA guideline as a screening tool was evaluated by calculating sensitivity and specificity for identifying patients in whom TTE would demonstrate major abnormality that would change management. Data were analyzed using SPSS (version 20.0; SPSS, Chicago, IL, USA). Statistical significance was defined as a value of $p<0.05$.

\section{Results}

In total, 324 elderly patients ( $\geq 65$-year old) with hip fracture were surgically treated in our hospital during the study period. After reviewing the electronic medical records, 43 patients were excluded ( 5 with multiple fractures, 6 with pathological fracture, 3 with peri-prosthetic fracture, 16 with unavailable operation rooms or anticoagulant withdrawal, 13 with cancer history), 281 patients met the inclusion and exclusion criteria finally. Of those cases included, 174 patients $(61.9 \%)$ underwent preoperative TTE, the rest 107 patients did not undergo the TTE. Seventy-one of the 174 patients (40.8\%) with preoperative TTE met the ACC/AHA guidelines, whereas 103 patients (59.2\%) received unnecessary TTE out of guidelines. Of the 104 patients who did not have TTE, 8 (7.4\%) met the ACC/AHA guideline (Figure. 1).

Further analysis of the patients who did not meet the ACC/AHA guidelines was conducted, comparing those who underwent TTE $(n=103)$ and those who did not $(n=99)$. There were no statistically significant differences in age, gender, or fracture or ASA class or anesthesiology type or treatment type. Most participants were female, and the most common fracture type was femoral neck. The majority of patients were treated using hemiarthroplasty (table 1).

As for preexisting medical conditions comparison, patients who underwent TTE had a significantly higher rate of chronic obstructive pulmonary disease $(17.5 \%$ vs. $6.1 \%, P=0.012)$. No significant differences were noted in in history of cardiovascular disease, diabetes or other comorbidities listed in table 1.

Among the 202 patients (103 with TTE and 99 without TTE) who did not meet the guidelines for preoperative TTE, those who received a preoperative TTE had a significantly longer average time to surgery (4.24 vs. 2.10 days, $P=0.000$ ) and significantly longer hospital length of stay (10.44 vs. 7.72 days, $P=0.000$ ) (table 2). Though there was higher rate of pressure sore in patients with TTE, there was no statistical differences. In addition, there were no significant differences in postoperative cardiac complications or others listed at table 3 . 
Additional analyses with the subgroup of patients with a preoperative TTE explored how frequently TTE reveals cardiac abnormality with potentiality of changing management and whether application of guidelines to indicate TTE was practicable or not. The rate of adherence to the ACC/AHA guideline was $40.8 \%(n=71)$. The most common indications for the TTE performed in adherence to the guidelines were known heart disease and over 1 year since the last TTE $(n=37)$, heart murmur $(n=17)$, known heart disease with worsened symptoms $(n=7)$, and dyspnea of unknown origin $(n=10)$. The rest of TTE was ordered for generic "evaluation of cardiac function" although the patient did not show related cardiac symptoms. After reviewing 174 TTE reports, 30 patients were identified by the TTE as having cardiac abnormality with the potentiality to alter perioperative management, including moderate-severe valvular disease $(n=13)$, pulmonary hypertension $(n=7)$, decline in ejection fraction $(n=5)$, moderate-severe pericardial effusion $(n=5)$. Only 4 patients were identified as having major cardiac abnormality among 103 patients who underwent TTE out of guidelines, whereas 26 patients were identified as having major cardiac abnormality among 71 patients whose TTE was adherent to ACC/AHA guideline. As a result, the guidelines were the accurate screening tool to identify patients with major cardiac abnormality with a calculated sensitivity of $86.7 \%$ and a specificity of $68.7 \%$ (Table 4 ).

\section{Discussion}

Though the ACC/AHA guidelines set up the standard algorithm for preoperative cardiac assessment in non-cardiac surgery, its adherent rate was not known. In this study, we confirmed that preoperative TTE in elderly patients with hip fracture is frequently not according to these guidelines in daily practice. A large number of patients are receiving unnecessary preoperative TTE without detecting the major cardiac abnormality. Although no significant difference in postoperative complications was identified, those patients with preoperative TTE out of guideline had the longer time to surgery and longer total hospital length of stay.

Based on the ACC/AHA guidelines, the patients who require noninvasive cardiac testing are those with active high-risk cardiac conditions. High risk patients might benefit from delaying hip fracture surgery to assess or clear the cardiac comorbidities[8]. However, delaying surgery due to unrecommended TTE could lead to worse outcome[13]. As our results showed, patients who had the TTE out of guidelines had higher prevalence of chronic obstructive pulmonary disease compared with the patients without TTE. However, there was no significant difference in cardiac related factors, including history of cardiovascular disease or ASA class. One possible explanation was that the indications for TTE were unclear and were rarely adherent to guidelines in clinical practice. Thus, a standardized protocol to determine which patients should undergo TTE was extremely valuable in clinical practice.

Unnecessary surgery delay $(\mathbb{4 8 \mathrm { h }})$ increases the perioperative complications and mortality[14]. Patients without preoperative TTE was closed to this target time; however, those with TTE out of guideline were delayed almost 2 days. Previous studies have also shown a growing concern with surgery delay due to preoperative cardiac test. Luttrell et al. evaluated the impact of preoperative TTE on the elderly hip fractures (131 patients with TTE, 563 patients without TTE). They concluded that the patients with 
preoperative TTE had obvious longer waiting time to surgery[15]. Harun et al. reviewed the effect of preoperative non-invasive cardiac test on hip fracture patients and found that further cardiac test led to a significant delay to surgery[16]. Cluett et al. compared the outcomes between the patients with cardiac evaluation besides electrocardiograph (22 patients) and the control group with only electrocardiograph (86 patients), which found the patients with further cardiac test had obvious surgery delay[17].

Therefore, we need to balance the benefits of TTE for preoperative cardiac risk assessment and the morbidity caused by surgery delay. High risk patients may benefit from delaying hip fracture surgery to undergo TTE and optimize the cardiac comorbidities. However, delaying surgery with a preoperative TTE that is not recommended or contributing may lead to worse outcome. Although ACC/AHA guideline had set up the criteria to determine which patients need preoperative TTE in non-cardiac surgery, its accuracy of acting as a screening tool to identify high risk patient with major cardiac abnormality was rarely studied. Our study demonstrated that the sensitivity of ACA/AHA guidelines for identifying patients who may have major cardiac abnormality with the potential to modify anesthesia or medical management was as high as $86.7 \%$ and the specificity was $68.7 \%$. Our results supported those of a similar study from Chris et al, they reviewed 100 patients with preoperative TTE and found $66 \%$ was in accordance with the guideline. The sensitivity and specificity of guideline for identifying patients who may have cardiac abnormality were $100 \%$ and $40 \%$.[18]

These guidelines were also proved to be effective to prevent the overuse of the other cardiac test. Stitgen et al. found that only $29 \%$ of geriatric patients with hip fracture who received a cardiology consultation had met the ACC/AHA guidelines and the consultation out of guideline did not change perioperative management but caused prominent surgery delay[19]. Smeets et al. conducted a retrospective study involving 388 patients and found the most frequent reason for incorrect preoperative cardiac screening was overscreening. In addition, the delay to surgery was increased by $9.9 \mathrm{~h}$ in the case of overscreening[20]. Recently, Smeets et al. published the prospective study involving 166 hip fracture patients and $87 \%$ of patients received preoperative cardiac screening in adherence to guideline, which was associated with a diminished use of preoperative resources[21].

To our best knowledge, there was only one study evaluating the correspondance between guideline and TTE utilization before[18]. However, they divided the patients into 2 groups (with TTE and without TTE) without further subdividing based on whether adhering to guidelines or not. The further subdivision could eliminate other unwanted distrubance, making the result difference more solely caused by TTE out of guideline. In addition, the pericardial effusion was not regarded as the abnormality with potentiality to change management, but it was proved to affect perioperative management in recent study[11]. Our study showed implementation of guidelines could improve the approriate usage of preoperative TTE test, reduce unecessary surgery delay. Several limitations should be noticed when interpreting these results. Firstly, surgery delay is multifactorial not solely caused by the TTE test, however we tried to minor the bias by excluding patients with surgery delay due to the unavailability of an operating room, surgeon or anticoagulant withdrawl. Secondary, evaluating whether TTE was adherent to guideline or not was subjective, but we decreased the bias by assessing the cases by two iddependant observers (X.P.C, Y.C.M) 
blinded to the TTE result and disscussed with the third observer(QT.L) when they have different opinions and drawed the conclusion finally. Thridly, we mainly focused on whether TTE identify major cardiac abnnormality which was proved to change management in previous literature, this may overlook the other infromations provided on TTE.

\section{Conclusion}

A majority of preoperative TTE were out of guideline and did not appear to identify cardiac abnormality or meaningfully influence management, while caused surgery delay and longer hospital stays. Our data suggested that implementation of the ACC/AHA guidelines in perioperatie management could reduce unecessary TTE without sacrificing the patient safety.

\section{Abbreviations}

TTE, transthoracic echocardiograph; ACC, American College of Cardiology Foundation; AHA, American Heart Association; TLS, time to surgery; LLS, length of hospital stay; ASA, American Society of Anesthesiologists

\section{Declarations}

Acknowledgements

Not applicable

Authors' contributions

.P.C, Y.C.M and Q.J.Z designed the study and modified and approved the final version of the manuscript. X.P.C wrote the first draft of the manuscript. Y.C.M, Z.T.D and Q.T.L collected the data. Q.T.L, Y.C.M and J.X.L analyzed and interpreted the data. Q.J.Z revised the manuscript.

Funding

This work was supported by grants from the Natural Science Foundation of Guangdong Province (2018A0303130206)

Ethics approval and consent to participate

The review board of the Guangdong Provincial People's Hospital approved the whole research plan.

Consent for publication

All authors reviewed the final version of the manuscript and approved it for submission.

Availability of data and materials 
The data and materials are available from the medical records department of Guangdong Provincial People's Hospital

Competing interests

The authors declare that they have no competing interests.

Author details

a Guangdong Provincial People's Hospital, Guangdong Academy of Medical Sciences; School of Medicine, South China University of Technology

b Shantou University Medical College, Shantou, China.

\section{References}

1. Hung WW, Egol KA, Zuckerman JD, Siu AL Hip fracture management: tailoring care for the older patient. Jama, 2012,307(20): p. 2185-94. https://doi,org/10.1001/jama.2012.4842

2. Brauer $C A$, Coca-Perraillon $M$, Cutler $D M$, Rosen $A B$ Incidence and mortality of hip fractures in the United States. Jama, 2009,302(14): p. 1573-9. https://doi,org/10.1001/jama.2009.1462

3. Cher EWL, Allen JC, Howe TS, Koh JSB Comorbidity as the dominant predictor of mortality after hip fracture surgeries. Osteoporosis international : a journal established as result of cooperation between the European Foundation for Osteoporosis and the National Osteoporosis Foundation of the USA, 2019. https://doi,org/10.1007/s00198-019-05139-8

4. Spurrier E, Wordsworth D, Martin S, Norris R, Parker MJ Troponin T in hip fracture patients: prognostic significance for mortality at one year. Hip international : the journal of clinical and experimental research on hip pathology and therapy, 2011,21(6): p. 757-61. https://doi,org/10.5301/hip.2011.8840

5. Taheriazam A, Saeidinia A, Keihanian F Total hip arthroplasty and cardiovascular complications: a review. Therapeutics and clinical risk management, 2018,14: p. 685-90.

https://doi,org/10.2147/tcrm.s155918

6. Pincus D, Ravi B, Wasserstein D, et al. Association Between Wait Time and 30-Day Mortality in Adults Undergoing Hip Fracture Surgery. Jama, 2017,318(20): p. 1994-2003.

https://doi,org/10.1001/jama.2017.17606

7. Shiga T, Wajima Z, Ohe Y Is operative delay associated with increased mortality of hip fracture patients? Systematic review, meta-analysis, and meta-regression. Canadian journal of anaesthesia $=$ Journal canadien d'anesthesie, 2008,55(3): p. 146-54. https://doi,org/10.1007/bf03016088

8. Fleisher LA, Fleischmann KE, Auerbach AD, et al. 2014 ACC/AHA guideline on perioperative cardiovascular evaluation and management of patients undergoing noncardiac surgery: executive summary: a report of the American College of Cardiology/American Heart Association Task Force on Practice Guidelines. Circulation, 2014,130(24): p. 2215-45.

https://doi,org/10.1161/cir.0000000000000105 
9. Grol R, Grimshaw J From best evidence to best practice: effective implementation of change in patients' care. Lancet (London, England), 2003,362(9391): p. 1225-30.

https://doi,org/10.1016/s0140-6736(03)14546-1

10. Canty DJ, Royse CF, Kilpatrick D, Williams DL, Royse AG The impact of pre-operative focused transthoracic echocardiography in emergency non-cardiac surgery patients with known or risk of cardiac disease. Anaesthesia, 2012,67(7): p. 714-20. https://doi,org/10.1111/j.13652044.2012.07118.x

11. Acan AE, Gultac E, Kilinc CY, et al. Preoperative Mild Pericardial Effusion Is Associated With Perioperative Complications In Elderly Patients Following Hip Fracture Surgery. Journal of investigative surgery : the official journal of the Academy of Surgical Research, 2019: p. 1-6. https://doi,org/10.1080/08941939.2019.1658832

12. Barber RL, Fletcher SN A review of echocardiography in anaesthetic and peri-operative practice. Part 1: impact and utility. Anaesthesia, 2014,69(7): p. 764-76. https://doi,org/10.1111/anae.12663

13. Bernstein J, Roberts FO, Wiesel BB, Ahn J Preoperative Testing for Hip Fracture Patients Delays Surgery, Prolongs Hospital Stays, and Rarely Dictates Care. Journal of orthopaedic trauma, 2016,30(2): p. 78-80. https://doi,org/10.1097/bot.0000000000000444

14. Simunovic N, Devereaux PJ, Sprague S, et al. Effect of early surgery after hip fracture on mortality and complications: systematic review and meta-analysis. CMAJ : Canadian Medical Association journal = journal de l'Association medicale canadienne, 2010,182(15): p. 1609-16. https://doi,org/10.1503/cmaj.092220

15. Luttrell K, Nana A Effect of Preoperative Transthoracic Echocardiogram on Mortality and Surgical Timing in Elderly Adults with Hip Fracture. Journal of the American Geriatrics Society, 2015,63(12): p. 2505-9. https://doi,org/10.1111/jgs.13840

16. Mutlu H, Bilgili F, Mutlu S, et al. The effects of preoperative non-invasive cardiac tests on delay to surgery and subsequent mortality in elderly patients with hip fracture. Journal of back and musculoskeletal rehabilitation, 2016,29(1): p. 49-54. https://doi,org/10.3233/bmr-150595

17. Cluett J, Caplan J, Yu W Preoperative cardiac evaluation of patients with acute hip fracture. American journal of orthopedics (Belle Mead, NJ), 2008,37(1): p. 32-6.

18. Adair C, Swart E, Seymour R, Patt J, Karunakar MA Clinical Practice Guidelines Decrease Unnecessary Echocardiograms Before Hip Fracture Surgery. The Journal of bone and joint surgery American volume, 2017,99(8): p. 676-80. https://doi,org/10.2106/jbjs.16.01108

19. Stitgen A, Poludnianyk K, Dulaney-Cripe E, Markert R, Prayson M Adherence to Preoperative Cardiac Clearance Guidelines in Hip Fracture Patients. Journal of orthopaedic trauma, 2015,29(11): p. 500-3. https://doi,org/10.1097/bot.0000000000000381

20. Smeets SJ, Poeze M, Verbruggen JP Preoperative cardiac evaluation of geriatric patients with hip fracture. Injury, 2012,43(12): p. 2146-51. https://doi,org/10.1016/j.injury.2012.08.007

21. Smeets SJM, van Wunnik BPW, Poeze M, Slooter GD, Verbruggen J Cardiac overscreening hip fracture patients. Archives of orthopaedic and trauma surgery, 2019. 
https://doi,org/10.1007/s00402-019-03270-z

\section{Tables}

Table 1. Patient characteristics of 202 patients who did not meet ACC/AHA guidelines 


\begin{tabular}{|c|c|c|c|}
\hline Characteristics & $\begin{array}{l}\text { With TTE } \\
(n=103)\end{array}$ & $\begin{array}{l}\text { Without TTE } \\
(n=99)\end{array}$ & $P$ value \\
\hline Age (years) & $81.07 \pm 6.08$ & $79.51 \pm 8.61$ & 0.140 \\
\hline Gender, n (\%) & & & 0.081 \\
\hline Female & $80(77.7)$ & $66(66.7)$ & \\
\hline Male & $23(22.3)$ & $33(33.3)$ & \\
\hline Fracture type, n (\%) & & & 0.597 \\
\hline Femoral neck & $57(55.3)$ & $57(57.6)$ & \\
\hline Intertrochanteric & $44(42.7)$ & $42(42.4)$ & \\
\hline Subtrochanteric & $2(1.9)$ & $0(1.1)$ & \\
\hline Treatment type, n (\%) & & & 0.457 \\
\hline Hemiarthroplasty & $41(39.8)$ & 33(33.3) & \\
\hline Total hip arthroplasty & 18 (17.5) & $21(21.2)$ & \\
\hline Proximal femoral nail anti-rotation & $44(42.7)$ & $43(43.5)$ & \\
\hline Cannulated screw fixation & $0(0)$ & $2(2)$ & \\
\hline ASA class, $n \llbracket \% \bigotimes$ & & & 0.848 \\
\hline ASA I & $23(22.3)$ & $24(24.2)$ & \\
\hline ASA II & $48(46.6)$ & $41(41.5)$ & \\
\hline ASA III & $28(27.2)$ & $31(31.3)$ & \\
\hline ASA IV & $4(3.9)$ & $3(3)$ & \\
\hline Anesthesiology type, n (\%) & & & 0.994 \\
\hline General anesthesia & $27(26.2)$ & $26(26.3)$ & \\
\hline Regional anesthesia & $76(73.8)$ & 73(73.7) & \\
\hline \multicolumn{4}{|l|}{ Preexisting medical history } \\
\hline Cardiovascular disease & 73(70.9) & $59(59.6)$ & 0.092 \\
\hline COPD & 18(17.5) & $6(6.1)$ & 0.012 \\
\hline Cerebrovascular disease & $20(19.4)$ & 15(15.2) & 0.423 \\
\hline Diabetes & $22(21.4)$ & 18(18.2) & 0.571 \\
\hline Previous fragility fracture & 15(14.6) & $7(7.1)$ & 0.087 \\
\hline
\end{tabular}


Abbreviations: TTE, transthoracic echocardiography; ASA, American Society of Anesthesiologists; COPD, Chronic obstructive pulmonary disease

Table 2. Treatment timing for 202 patients who did not meet guidelines

\begin{tabular}{|llll|}
\hline Treatment time & $\begin{array}{l}\text { Non-TTE } \\
\nabla n=99 \unrhd\end{array}$ & $\begin{array}{l}\text { TTE } \\
\Downarrow n=103 \rrbracket\end{array}$ & P value \\
\hline Length of hospital stay, days & $7.72 \pm 3.24$ & $10.44 \pm 4.56$ & 0.000 \\
\hline Time to surgery, days & $2.10 \pm 2.13$ & $4.24 \pm 2.11$ & 0.000 \\
\hline Time from surgery to discharge, days & $5.68 \pm 2.60$ & $6.19 \pm 3.66$ & 0.256 \\
\hline
\end{tabular}

Abbreviations: TTE, transthoracic echocardiography.

Table 3. Comparison of postoperative complications for patients who did not meet the guideline

\begin{tabular}{|llll|}
\hline Postoperative complications & $\begin{array}{l}\text { Non-TTE (\%) } \\
\mathrm{n}=99\end{array}$ & $\begin{array}{l}\text { TTE (\%) } \\
\mathrm{n}=103\end{array}$ & P Value \\
\hline New onset malignant arrhythmia & $3(3.0)$ & $8(7.8)$ & 0.138 \\
\hline Acute myocardial infarction & $2(2.0)$ & $3(2.9)$ & 1.000 \\
\hline Heart failure & $3(3.0)$ & $5(4.9)$ & 0.721 \\
\hline Pneumonia & $3(3.0)$ & $6(5.8)$ & 0.499 \\
\hline Acute kidney failure & $0(0)$ & $1(0.9)$ & 1.000 \\
\hline Cerebrovascular accident & $3(3.0)$ & $5(4.9)$ & 0.721 \\
\hline Surgical site infection & $2(2.0)$ & $5(4.9)$ & 0.474 \\
\hline Pressure sores & $2(2.0)$ & $7(6.8)$ & 0.100 \\
\hline Delirium & $8(8.1)$ & $10(9.7)$ & 0.685 \\
\hline Intensive care unit stay & $2(2.0)$ & $0(0)$ & 0.239 \\
\hline
\end{tabular}

Abbreviations: TTE, transthoracic echocardiography. 
Table 4. Result about whether the TTE met the guidelines and the TTE results reviewing

\begin{tabular}{|lll|}
\hline \multicolumn{3}{|c|}{ TTE results reviewing } \\
& With major abnormality & Without major abnormality \\
\hline Guideline criteria & & \\
\hline Adhere to guideline & 26 & 45 \\
\hline Out of guideline & 4 & 99 \\
\hline
\end{tabular}

\section{Figures}

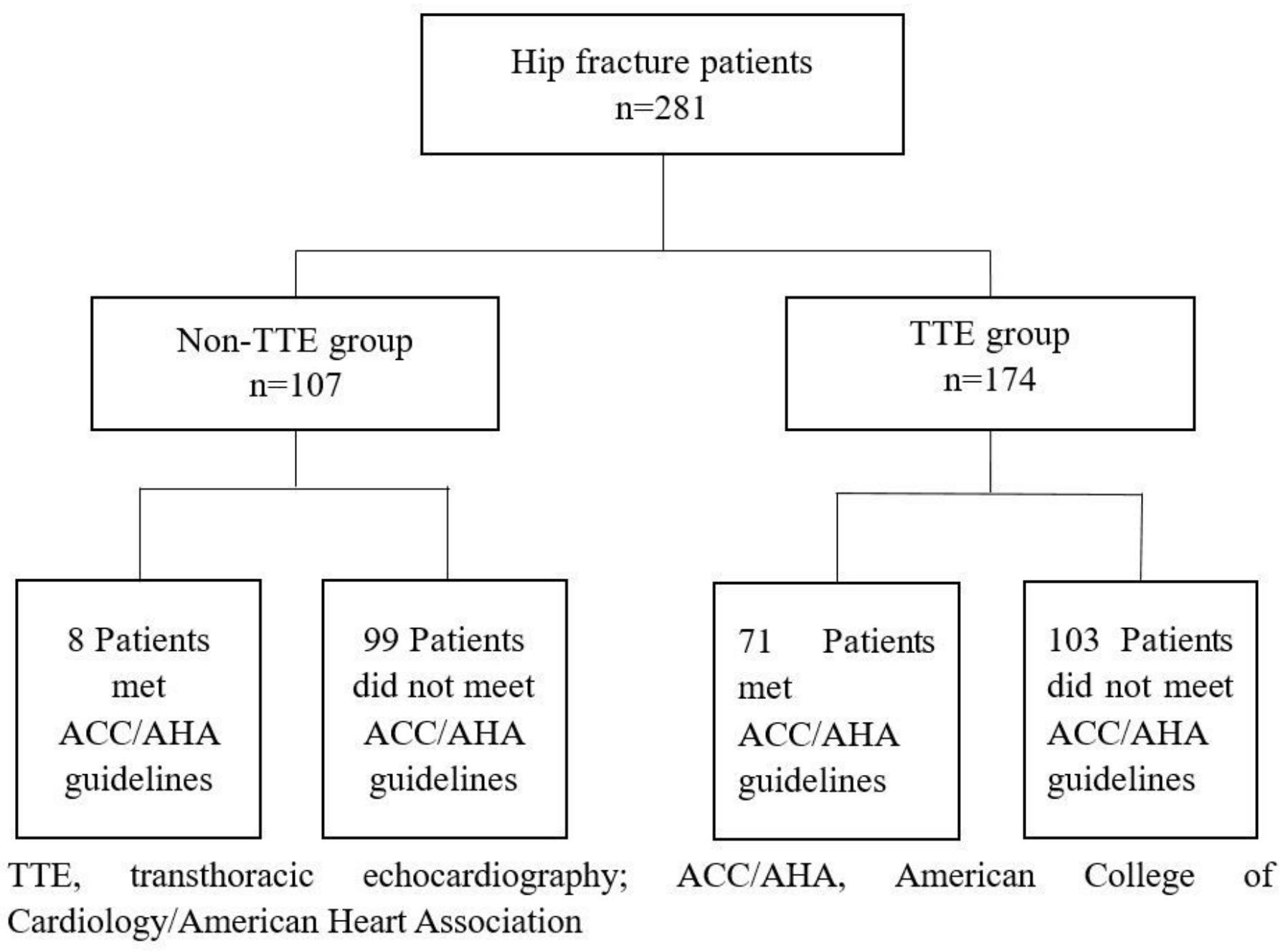

Figure 1

Preoperative TTE and ACC/AHA guidelines status for 281 hip fracture patients. 